\title{
Differential Form Approach for Stationary Axisymmetric Maxwell Fields in General Relativity
}

\author{
L. Fernández-Jambrina and F.J. Chinea \\ Departamento de Física Teórica II, \\ Facultad de Ciencias Físicas, \\ Universidad Complutense \\ 28040-Madrid, Spain
}

October 14, 2013

\begin{abstract}
A formulation for stationary axisymmetric electromagnetic fields in general relativity is derived by casting them into the form of an anisotropic fluid. Several simplifications of the formalism are carried out in order to analyze different features of the fields, such as the derivation of electromagnetic sources for the Maxwell field in the form of thin layers, construction of new solutions, and generation techniques.

PACS: 04.20.Cv, 04.20.Jb, 04.40.+c
\end{abstract}

\section{Introduction}

The study of coupled stationary axisymmetric electromagnetic and gravitational fields is of great interest in astrophysics in order to provide a description for the exterior of steadily rotating objects. Although the whole system of equations can be reduced to two complex partial differential equations [1] and special techniques have been developed to generate solutions, for instance the ones reviewed in [2], [3] for vacuum fields, we still lack exact solutions whose physical interpretation is appealing.

In section 2 we shall extend the exterior differential system formalism presented in [4] and [5] for perfect fluids to electromagnetic fields. The Maxwell field will be presented as an anisotropic fluid. Null fields will not be considered since they have a null Killing vector [6] and have already been studied. The main advantage of using differential forms is that we are not forced to choose coordinates from the beginning and therefore there is more freedom for solving 
the equations and simplifying the system in different ways. In section 3 a simplification will be carried out on this Maxwell fluid to cast it into a shear-free form. Advantage will be taken from it to construct magnetic dipole thin layers as surface sources for the magnetic field of static (among other) electrovacs and to extend previous results on angular momentum densities for vacuum metrics to some electrovacs. Section 4 is devoted to another simplification of the formulation. This time the Maxwell fluid will take the form of an irrotational fluid. An extension of some results obtained in canonical coordinates is provided. In section 5 it is performed a simplification of different nature: The Ricci tensor is diagonalized and a family of new electrovacs is constructed.

\section{Exterior differential system for stationary ax- isymmetric electrovacs}

\subsection{Cartan's first structure equations}

We shall follow the approach developed in [4] and [5] for the kinematical quantities and define an orthonormal vierbein $\left\{\theta^{0}, \theta^{1}, \theta^{2}, \theta^{3}\right\}$ where $\left\{\theta^{0}, \theta^{1}\right\}$ lie on the space spanned by the orbits of the isometries $\left\{\partial_{t}, \partial_{\phi}\right\}$ whereas $\left\{\theta^{2}, \theta^{3}\right\}$ will lie on the orthogonal space. In order to partially diagonalize the electromagnetic stress tensor we shall choose $\theta^{0}$ parallel to the direction of its timelike eigenvector as the timelike leg of the tetrad. The four eigenvalues of the stress tensor have the same absolute value and one of them is negative. This one corresponds generically to the eigenvector which lies in the direction of the magnetic and electric field in a frame in which they are parallel or, if the Lorentz invariant $E \cdot B$ is zero, then the respective eigenvector lies in the direction of the electric (magnetic) field in a frame in which the magnetic (electric) field vanishes. Therefore we shall be able to view the electromagnetic field as an anisotropic fluid with the absolute value of the pressure equal to the density and consider $u=-\theta^{0}$ as its velocity form. Imposing that the tetrad be torsion-free we get the following Cartan equations:

$$
\begin{gathered}
d u=a \wedge u+w \wedge \theta^{1} \\
d \theta^{1}=(b-a) \wedge \theta^{1}+s \wedge u \\
d \theta^{2}=-\nu \wedge \theta^{3} \\
d \theta^{3}=\nu \wedge \theta^{2}
\end{gathered}
$$

where $a$ is the acceleration of the 'fluid', $w=* \omega$ ( $\omega$ is the vorticity form derived from $u$ and $*$ stands for the two-dimensional Hodge dual in the space orthogonal to the orbits of the Killing vectors $\left.\left\{\partial_{t}, \partial_{\phi}\right\}\right), s$ is a one-form associated with the shear of the fluid, $b=d \ln \rho$ ( $\rho$ is the radial pseudocylindrical Weyl coordinate) and $\nu$ is just a connection in the two-space spanned by $\theta^{2}$ and $\theta^{3}$. We still have a great amount of freedom for further simplifications. 


\subsection{Bianchi identities}

Bianchi identities are easily obtained by exterior differentiation of the previous set of equations:

$$
\begin{gathered}
d b=0 \\
d a=w \wedge s \\
d w=-(b-2 a) \wedge w \\
d s=(b-2 a) \wedge s
\end{gathered}
$$

\subsection{Maxwell's equations}

Now we shall describe the Faraday and Maxwell electromagnetic strength twoforms ( $F$ and its four-dimensional dual ${ }^{4} * F$ ) in terms of the electric and magnetic one-forms, respectively $E$ and $B$ ). In what follows we shall restrict ourselves to electromagnetic forms $E$ and $B$ with no projection on the orbits of the Killing vectors:

$$
\begin{gathered}
F=-E \wedge u+* B \wedge \theta^{1} \\
{ }^{4} * F=B \wedge u+* E \wedge \theta^{1}
\end{gathered}
$$

Therefore, the Maxwell vacuum equations $d F=0$ and $d^{4} * F=0$ imply:

$$
\begin{gathered}
d E-E \wedge a+* B \wedge s=0 \\
d * B+E \wedge w-* B \wedge(b-a)=0 \\
d * E-B \wedge w-* E \wedge(b-a)=0 \\
d B-B \wedge a-* E \wedge s=0
\end{gathered}
$$

These equations are not a consequence of the integrability conditions for the Einstein equations unless the Lorentz scalar $E \cdot B$ is different from zero. These equations can be written in a more compact expression defining a complex oneform $f$ :

$$
\begin{gathered}
f=E+i B \\
d f=-a \wedge f-i s \wedge * f \\
d * f=(a-b) \wedge * f+i w \wedge f
\end{gathered}
$$




\subsection{Einstein's equations}

Considering that the electromagnetic stress tensor has the following expression:

$$
T_{b}^{a}=\frac{1}{4 \pi}\left\{F^{a c} F_{b c}-\frac{1}{4} \delta_{b}^{a} F^{c d} F_{c d}\right\}
$$

the Einstein equations take the general form:

$$
\begin{gathered}
d *(w-s)+2 a \wedge * w+2(a-b) \wedge * s=4 E \wedge B=2 i f \wedge \bar{f} \\
d * a+b \wedge * a+\frac{1}{2} w \wedge * w-\frac{1}{2} s \wedge * s=E \wedge * E+B \wedge * B=\bar{f} \wedge * f \\
d * b+b \wedge * b=0 \\
d \tilde{b}+b \wedge \tilde{b}-\frac{1}{2}(s-w) \wedge(\tilde{s}-\tilde{w})+2 a \wedge \tilde{a}-2 b \wedge \tilde{a}+ \\
+2 \nu \wedge * \tilde{b}=2 E \wedge \tilde{E}+2 B \wedge \tilde{B}=2 \bar{f} \wedge \tilde{f} \\
d * \tilde{b}+b \wedge * \tilde{b}-\frac{1}{2}(s-w) \wedge *(\tilde{s}-\tilde{w})+2 a \wedge * \tilde{a}-2 b \wedge * \tilde{a}- \\
-2 \nu \wedge \tilde{b}=2 E \wedge * \tilde{E}+2 B \wedge * \tilde{B}=2 \bar{f} \wedge * \tilde{f} \\
d \nu+a \wedge * b-a \wedge * a+\frac{1}{4}(s-w) \wedge *(s-w)=0
\end{gathered}
$$

The symbol $\sim$ denotes the linear transformation in the $\left\{\theta^{2}, \theta^{3}\right\}$-space defined by the following expression:

$$
\tilde{\theta}^{2}=\theta^{2} \quad \tilde{\theta}^{3}=-\theta^{3}
$$

As in the perfect fluid case, equations 22 to 24 can be left for the end, since the connection $\nu$ can be algebraically obtained from them and then equation 24 is automatically satisfied.

\section{The electromagnetic field as a rigidly rotating fluid}

\subsection{Ernst's equations}

As we have already said, our exterior system can be simplified in many ways. This freedom comes from the possibility of describing the electromagnetic field in different reference frames preserving the structure of the equations. We can shift

from one frame to another performing a local boost or rotation or a combination of both. 
One boost that can always be applied is the one which takes us from our original frame to another in which the velocity $u$ is shear-free $(s=0)$. This transformation is described by the following equations:

$$
\begin{gathered}
u^{\prime}=\cosh \lambda u+\sinh \lambda \theta^{1} \\
\theta^{1^{\prime}}=\sinh \lambda u+\cosh \lambda \theta^{1} \\
d \lambda=\cosh \lambda \sinh \lambda\left(b^{\prime}-2 a^{\prime}\right)+\cosh ^{2} \lambda s^{\prime}-\sinh ^{2} \lambda w^{\prime}
\end{gathered}
$$

The electromagnetic field changes in the following way:

$$
\begin{aligned}
& E=\cosh \lambda E^{\prime}-\sinh \lambda * B^{\prime} \\
& B=\cosh \lambda B^{\prime}+\sinh \lambda * E^{\prime}
\end{aligned}
$$

This transformation is compatible with the whole set of equations as it happened in the vacuum case [4]. Therefore there is no loss of generality in taking $s=0$ from the beginning.

We can formally integrate equations 5 to 7 and 16 to get:

$$
\begin{gathered}
a=d U \quad b=d \ln \rho \\
w=\rho^{-1} e^{2 U} d A \\
f=-e^{-U} d \Phi
\end{gathered}
$$

From equation 19 we obtain the exact differential that defines the twist potential $\chi[1]$ :

$$
d \chi=e^{2 U} * w+2 i \bar{\Phi} d \Phi
$$

and construct the Ernst potential $\varepsilon$ in the following way:

$$
d \varepsilon=d e^{2 U}+i d \chi \quad \mathcal{R} \varepsilon=e^{2 U}-\bar{\Phi} \Phi
$$

Substituting 33 in 17 we get an elliptic equation for the scalar complex electromagnetic potential $\Phi$ :

$$
d * d \Phi+b \wedge * d \Phi=\frac{1}{\mathcal{R} \varepsilon+\Phi \bar{\Phi}}\{d \varepsilon+2 \bar{\Phi} d \Phi\} \wedge * d \Phi
$$

And if we combine the previous equation with the Raychaudhuri equation (20), and 34 with 31 and 7, we obtain another second order elliptic equation, namely the Ernst equation:

$$
d * d \varepsilon+b \wedge * d \varepsilon=\frac{1}{\mathcal{R} \varepsilon+\Phi \bar{\Phi}}\{d \varepsilon+2 \bar{\Phi} d \Phi\} \wedge * d \varepsilon
$$


The whole set of equations for stationary axisymmetric electrovacs reduces to these two complex equations for the potentials $\varepsilon$ and $\Phi$ [1].

The equations 5 and 21 just introduce two functions $\rho$ and $z$, the Weyl pseudocylindrical coordinates, that are related by the Hodge dual of their differentials:

$$
* d \rho=-d z
$$

And therefore the metric takes the following form, after integrating the Cartan equations:

$$
d s^{2}=-e^{2 U}(d t-A d \phi)^{2}+e^{-2 U}\left[e^{2 k}\left(d \rho^{2}+d z^{2}\right)+\rho^{2} d \phi^{2}\right]
$$

\subsection{Construction of electromagnetic dipole surface densi- ties}

This formalism can be used to obtain electric and magnetic dipole surface densities for the sources of some asymptotically flat stationary axially symmetric solutions of the Einstein-Maxwell equations in a similar way as it was done in [7] to construct angular momentum densities for vacuum metrics. The formulae derived by Israel in [8] cannot be applied since they only yield non-vanishing expressions for the mass, charge and angular momentum, but not for the magnetic momentum.

We shall only consider static fields and also nonstatic metrics for which $w \wedge f=0$-if we want to calculate only magnetic (electric) moment densities, we shall only need $w \wedge E=0 \quad(w \wedge B=0)$-. By asymptotically flat we mean that the metric has the following behavior at infinity $(r \rightarrow \infty)$ in some coordinates $(t, r, \theta, \phi)$ :

$$
\begin{array}{r}
d s^{2}=-\left(1-\frac{2 m}{r}\right)\left(d t+\frac{2 J \sin ^{2} \theta}{r} d \phi\right)^{2}+ \\
+\left(1+\frac{2 m}{r}\right)\left[d r^{2}+r^{2}\left(d \theta^{2}+\sin ^{2} \theta d \phi^{2}\right)\right]
\end{array}
$$

where $m$ is the source's total mass and $J$ is the total angular momentum. Therefore the metric functions $e^{2 U}$ and $A$ and the twist potential $\chi$ (since $A$ and $\chi$ are related by the dual of the vorticity one-form) take this form at infinity:

$$
\begin{gathered}
e^{2 U}=1-\frac{2 m}{r}+O\left(\frac{1}{r^{2}}\right) \quad A=-\frac{2 J \sin ^{2} \theta}{r}+O\left(\frac{1}{r^{2}}\right) \\
\chi=-\frac{2 J \cos \theta}{r^{2}}+O\left(\frac{1}{r^{3}}\right)
\end{gathered}
$$


To calculate the magnetic dipole we shall require that the magnetic scalar potential, that is the imaginary part $V$ of the potential $\Phi$ must be asymptotically dipolar:

$$
V=\frac{M \cos \theta}{r^{2}}+O\left(\frac{1}{r^{3}}\right)
$$

As it happens in flat spacetime, the magnetic scalar potential can be defined only outside the sources. If we have a sheet of dipoles as a source, the limit values of the potential on either side of the surface will be different and this discontinuity reveals the existence of the source (cfr. for instance [9]). Our purpose will be to extend this result to curved spacetimes.

Following the approach developed in [7], we integrate equation 12 with the previously stated restrictions, that combined with 33 provides us with two expressions for the magnetic field:

$$
\begin{gathered}
B=\mathcal{I}\{f\}=-e^{-U} d V=-\rho^{-1} e^{U} * d W \\
V=\mathcal{I}\{\Phi\}
\end{gathered}
$$

This function $W$ has the following asymptotic expression due to the behavior of $V$ at infinity:

$$
W=\frac{M \sin ^{2} \theta}{r}+O\left(\frac{1}{r^{2}}\right)
$$

And now we shall integrate the scalar product of the magnetic field $B$ and the differential of a function $Z(r, \theta)$ to be determined. The domain of integration will be the space $V_{3}$ orthogonal to the congruence defined by $u$. The metric on this space is the projection $g={ }^{4} g+u \otimes u$. Using both expressions for $B$ we get:

$$
\begin{array}{r}
0=\int_{V_{3}} \sqrt{g}<[B+*(* B)], d Z>d x^{1} d x^{2} d x^{3}= \\
=\int_{V_{3}} \sqrt{g}\left\{-e^{-U} g^{\mu \nu} \partial_{\mu} V \partial_{\nu} Z+\rho^{-1} e^{U} \varepsilon^{\mu \nu} \partial_{\mu} W \partial_{\nu} Z\right\} d x^{1} d x^{2} d x^{3}
\end{array}
$$

In order to express the integrand as a total derivative we choose $Z$ so that it fulfills the following differential equation:

$$
\partial_{\mu}\left(\sqrt{g} e^{-U} g^{\mu \nu} \partial_{\nu} Z\right)=0
$$

As an asymptotic boundary condition we impose that $Z$ behaves like $r \cos \theta$ at infinity.

The potential $V$ is discontinuous across a closed surface $S$ if we are to have a magnetic moment surface density, therefore we have to split the space into two 
pieces $V_{3}^{+}$and $V_{3}^{-}$, respectively the outer and inner part of $V_{3}$. If the surface $S$ is open, it can be extended to a closed one, taking the value zero for the discontinuity on the closure. $V^{+}$and $V^{-}$shall denote the limit values of $V$ on either side of the surface.

Since the Levi-Cività tensor on the space orthogonal to the orbits of the Killing fields is $\varepsilon^{\mu \nu}=e^{2(U-k)}[\mu \nu]$ the integrand in 47 can be written as:

$$
\partial_{\mu}\left\{-\sqrt{g} e^{-U} g^{\mu \nu} V \partial_{\nu} Z+W[\mu \nu] \partial_{\nu} Z\right\}
$$

We have the desired total derivative and we can express the integral as a surface integral on the boundary $\Sigma=\partial V_{3}^{+} \cup \partial V_{3}^{-}$with unitary normal $n$. The boundary of $V_{3}^{+}$consists of $S$ and the sphere at infinity $S^{2}(\infty)$ and the boundary of $V_{3}^{-}$is just $S$. Taking into account the required behavior of the metric functions and the magnetic potential at infinity, the integral on $S^{2}(\infty)$ yields:

$$
\int_{S} d S[V] e^{-U} g^{\mu \nu} n_{\mu} \partial_{\nu} Z=4 \pi M
$$

And therefore we obtain the following formula relating the jump of the magnetic potential $[V]$ across $S$ with the total magnetic moment $M$ :

$$
\begin{gathered}
4 \pi M=\int_{S} d S \sigma \\
\sigma=\frac{1}{4 \pi}[V] e^{-U} g^{\mu \nu} n_{\mu} \partial_{\nu} Z
\end{gathered}
$$

We can consequently infer that $\sigma$ is the source's magnetic moment density for the Maxwell field.

Similar calculations can be done with the real part of the electromagnetic potential $\Phi$ to compute electric dipole surface densities.

\subsection{Angular momentum surface densities}

The formalism developed in [7] for interpreting discontinuities of the twist potential of asymptotically flat stationary axially symmetric vacuum metrics can be extended in a straightforward way to cope with electrovac solutions with $E \wedge B=0$, since the only equations involved are the Bianchi equations and the equation for the vorticity, 19, and these remain the same as in the vacuum case if we impose the mentioned restriction. Hence the angular momentum surface density for stationary axisymmetric vacuum metrics and electrovacs with parallel magnetic and electric fields has the following expression in terms of the jump $[\chi]$ of the twist potential:

$$
\sigma_{r o t}=-\frac{1}{8 \pi}[\chi] e^{-3 U} g^{\mu \nu} n_{\mu} \partial_{\nu} Z_{r o t}
$$


where the function $Z_{r o t}$ is asymptotically $r \cos \theta$ and satisfies the following differential equation:

$$
\partial_{\mu}\left(\sqrt{g} e^{-3 U} g^{\mu \nu} \partial_{\nu} Z_{r o t}\right)=0
$$

\subsection{Bonnor's transformation}

One of the peculiarities of this formalism for constructing surface densities is its behavior under Bonnor transformations. These transformations are a method for generating magnetostatic electrovacs from stationary nonstatic metrics [10]. As the solutions of the Einstein-Maxwell equations are fully characterized by the Ernst and electromagnetic potentials, it will suffice to give the rules that determine the potentials for the electrovac:

$$
\begin{gathered}
\varepsilon=\varepsilon_{v a c} \bar{\varepsilon}_{v a c} \\
V=i \chi_{v a c}
\end{gathered}
$$

In order to have a real solution the magnetic potential has to be real, therefore the parameters have to be rearranged with a complex transformation to achieve this purpose.

From these expressions it is clear that $e^{U}=e_{v a c}^{2 U}$. Since $Z$ is a function only of $\rho$ and $z$, in canonical coordinates, the only components of the three-metric $g$ of $V_{3}$ that appear in the differential equation 48 are $g^{\rho \rho}=g^{z z}=e^{2 U-2 k}$. The square root of the determinant of this metric is $\sqrt{g}=e^{-3 U+2 k} \rho$ and therefore equation 48 takes this form in terms of the original vacuum metric functions after rearranging the parameters:

$$
\begin{aligned}
\partial_{\mu}\left(\sqrt{g} e^{-U} g^{\mu \nu} \partial_{\nu} Z\right) & =\partial_{\mu}\left(e^{-2 U} \rho \delta_{\mu \nu} \partial_{\nu} Z\right)= \\
\partial_{\mu}\left(e_{v a c}^{-4 U} \rho \delta_{\mu \nu} \partial_{\nu} Z\right) & =\partial_{\mu}\left(\sqrt{g_{v a c}}\left(e^{-3 U}\right)_{v a c} g_{v a c}^{\mu \nu} \partial_{\nu} Z\right)=0
\end{aligned}
$$

That is, the function $Z$ needed for the construction of the magnetic dipole surface density satisfies the same differential equation as the $Z_{\text {rot }}$ involved in the calculation of the angular momentum density for the original vacuum metric

$g_{v a c}$. Therefore the same function is valid for both solutions, once we have reinterpreted the parameters, and the formalism is compatible with the Bonnor transformation.

\subsection{An example: Bonnor's massive magnetic dipole}

As an example of how this formalism works, we shall calculate the magnetic source for Bonnor's massive magnetic dipole [11]. This is a Bonnor transformation of the Kerr metric and therefore we can use the $Z$ function obtained in [7] for its source's angular momentum density. 
Bonnor's metric has the following form:

$$
\begin{array}{r}
d s^{2}=-\left(1-\frac{2 m r}{r^{2}-a^{2} \cos ^{2} \theta}\right)^{2} d t^{2}+ \\
+\left(1-\frac{2 m r}{r^{2}-a^{2} \cos ^{2} \theta}\right)^{-2}\left\{\left(r^{2}-a^{2}-2 m r\right) \sin ^{2} \theta d \phi^{2}+\right. \\
\left.\frac{\left(r^{2}-a^{2} \cos ^{2} \theta-2 m r\right)^{4}}{\left[(r-m)^{2}-\left(a^{2}+m^{2}\right) \cos ^{2} \theta\right]^{3}}\left(d \theta^{2}+\frac{d r^{2}}{r^{2}-2 m r-a^{2}}\right)\right\}
\end{array}
$$

from which we can read the necessary metric functions:

$$
e^{2 U}=\left(1-\frac{2 m r}{r^{2}-a^{2} \cos ^{2} \theta}\right)^{2}=1-\frac{4 m}{r}+0\left(\frac{1}{r^{2}}\right)
$$

The magnetic field is determined by the scalar potential:

$$
V=\frac{2 a m \cos \theta}{r^{2}-a^{2} \cos ^{2} \theta}=\frac{2 a m \cos \theta}{r^{2}}+0\left(\frac{1}{r^{4}}\right)
$$

Therefore both the gravitational and magnetic fields fulfill the required asymptotic conditions with mass equal to $2 \mathrm{~m}$ and magnetic moment $2 \mathrm{am}$.

If we consider the metric to be written in a sort of oblate spheroidal coordinates as it is done in [8] for the Kerr metric, then events on the surface $r=0$ with polar angle $\theta$ have to be identified with events with polar angle equal to $\pi-\theta$. To avoid double-counting these points, the $\theta$ coordinate will range from 0 to $\pi / 2$ on this surface. Therefore the function $\cos \theta$ suffers a discontinuity upon crossing $r=0$, since it shifts sign from positive in the upper subspace $(r>0$, $0 \leq \theta<\pi / 2)$ to negative in the lower subspace $(r>0, \pi / 2 \leq \theta<\pi)$.

Hence the magnetic potential $V=\mathcal{I}\{\Phi\}$ is discontinuous on the surface $r=0$. The difference between the values taken on the upper and the lower side of the surface is:

$$
[V]=-\frac{4 m}{a \cos \theta}
$$

The $Z$ function satisfying 48 can be obtained from the $Z_{\text {rot }}$ for Kerr, as it was shown in the previous section, taking into account that $a_{K e r r}=i a$. Hence,

$$
Z=(r-3 m) \cos \theta-\frac{2 a^{2} m\left(5 \cos ^{3} \theta-3 \cos \theta\right)}{5\left(r^{2}-a^{2} \cos ^{2} \theta\right)}
$$

The surface element for $r=0$ is:

$$
d S=\frac{a^{5} \sin \theta \cos ^{4} \theta}{\left|a^{2} \cos ^{2} \theta-m^{2} \sin ^{2} \theta\right|^{3 / 2}} d \theta d \phi
$$

We are ready now to write the magnetic moment surface density for the source of Bonnor's magnetic dipole using the formula 52: 


$$
\sigma=\frac{m}{\pi a^{4}} \frac{\left|a^{2} \cos ^{2} \theta-m^{2} \sin ^{2} \theta\right|^{3 / 2}}{\cos ^{4} \theta}
$$

The integral defining the total magnetic moment yields the expected result:

$$
M=\int_{S} \sigma d S=\int_{0}^{2 \pi} \int_{0}^{\pi / 2} d \theta(m a \sin \theta)=2 m a
$$

But unfortunately the source lies in a region where the signature of the metric is not the usual one (for instance, the angle $\phi$ is no longer a spacelike coordinate). Any other surface source would have to include the region $S$ in order to cope with the discontinuities of the magnetic potential. Therefore its interpretation as a physical material source is dubious and one would have to resort to three-dimensional sources to hide the unphysical regions of the electrovac metric.

\section{The electromagnetic field as an irrotational fluid}

\subsection{Ernst's equations}

Instead of performing a boost in order to cancel the shear one-form $s$, as it was done in the previous section, we can always write our equations in a frame where the dual of the vorticity $w$ is zero. This is also compatible with our system of equations, as it happened for vacuum fields [4], [5]. Of course, this would not be possible in general for a perfect fluid. The differential equation defining the $\lambda$ parameter of the boost is now:

$$
d \lambda=\cosh \lambda \sinh \lambda\left(2 a^{\prime}-b^{\prime}\right)+\cosh ^{2} \lambda w^{\prime}-\sinh ^{2} \lambda s^{\prime}
$$

This amounts to many simplifications, as it happened in the shear-free formalism. The equations 5 to 8 and 17 can be integrated to get:

$$
\begin{gathered}
a=d U \quad b=d \ln \rho \\
s=\rho e^{-2 U} d C \\
* f=-e^{U} \rho^{-1} d \Psi
\end{gathered}
$$

From equation 19 we get a exact differential that can be used to define what could be called 'deformation potential' $\psi$ in analogy with the twist potential:

$$
d \psi=\rho^{2} e^{-2 U} * s-2 i \bar{\Psi} d \Psi
$$


and construct another complex potential $\eta$ similar to the Ernst potential:

$$
d \eta=d\left(-\rho^{2} e^{-2 U}\right)-i d \psi \quad \mathcal{R}\{\eta\}=-\rho^{2} e^{-2 U}-\bar{\Psi} \Psi
$$

If we substitute 69 in 16 another generalization of the Laplace equation is achieved, this time for the potential $\Psi$ :

$$
d * d \Psi+b \wedge * d \Psi=\frac{1}{\mathcal{R} \eta+\Phi \bar{\Phi}}\{d \eta+2 \bar{\Psi} d \Psi\} \wedge * d \Psi
$$

The analog of the Ernst equation can be obtained in this irrotational formalism in a similar way taking into account the latter equation, the Raychaudhuri equation (20) and the equations 70, 67 and 8:

$$
d * d \eta+b \wedge * d \eta=\frac{1}{\mathcal{R} \eta+\Psi \bar{\Psi}}\{d \eta+2 \bar{\Psi} d \Psi\} \wedge * d \eta
$$

These two equations, together with the coordinate condition $* d \rho=d z$, comprise the whole problem of calculating stationary axially symmetric electrovac solutions. In this formalism the metric takes a form different from the canonical one:

$$
d s^{2}=-e^{2 U} d t^{2}+e^{-2 U}\left[e^{2 k}\left(d \rho^{2}+d z^{2}\right)+\rho^{2}(d \phi+C d t)^{2}\right]
$$

Both sets of equations, the Ernst equations (36 and 37) and these new Ernstlike equations, look the same exchanging $\varepsilon$ for $\eta$ and $\Phi$ for $\Psi$ as it happened in the vacuum case [4], [5]. However, there is a difference that did not occur for vacui and that is hidden behind the minus sign that it is written before the norm of the rotation Killing vector, $\left\|\partial_{\phi}\right\|=\rho^{2} e^{-2 U}$, as it appears in the definition for the $\eta$ potential. This sign enforces the real part of $\eta$ to be negative if it has to deal with a physical situation. Otherwise the rotation Killing vector would be timelike. On the other hand $\varepsilon$ can be either positive or negative. Besides, the conditions on the potentials for the metric to be asymptotically flat are rather different:

$$
\begin{gathered}
\varepsilon=1+0\left(\frac{1}{r}\right) \\
\eta=-r^{2} \sin ^{2} \theta+0(r)
\end{gathered}
$$

Although the equations have been cast in the same form, in fact the potentials have very different meaning.

One could think of the following transformation in order to generate new stationary axially symmetric electrovacs as the electromagnetic generalization of the transformation introduced in [4] and [5]:

$$
\varepsilon \longrightarrow \eta
$$




$$
\Phi \longrightarrow \Psi
$$

But this would lead to unphysical metrics unless the seed metric has (+---) signature, since this transformation induces the following exchange between the norms of the Killing vectors:

$$
e^{2 U} \longrightarrow-\rho^{2} e^{-2 U}
$$

It could be used then to generate new solutions from the forbidden regions of other spacetimes.

\subsection{Two families of 'irrotational' solutions}

The first attempt for constructing new solutions of the Einstein-Maxwell equations with the irrotational formalism could be to make use of the ansätze that have been profitable for solving the standard Ernst equation. For a start it can be shown that the Weyl family of static electrovacs [12] can be extended to yield new solutions.

The Weyl ansatz, in terms of the Ernst potentials, assumes a functional dependence between $\varepsilon$ and $\Phi$, that now are real (Since the metric is static, the twist potential can be taken to be null):

$$
\varepsilon=\varepsilon(\Phi)
$$

This ansatz leads to two equations, that can be integrated, considering the asymptotic flatness condition for the Ernst potential (75):

$$
\ddot{\varepsilon}(\Phi)=0 \Rightarrow e^{2 U}=1-2 c \Phi+\Phi^{2}
$$

where $c$ is a constant.

$$
d * d \Phi+b \wedge * d \Phi=\frac{-2 c+2 \Phi}{1-2 c \Phi+\Phi^{2}} d \Phi \wedge * d \Phi
$$

that can be solved in terms of a function $Y$, solution of the reduced Laplace equation:

$$
d * d Y+b \wedge * d Y=0
$$

via the following relation:

$$
\Phi=\left\{\begin{array}{l}
-\sqrt{c^{2}-1} \operatorname{coth} Y+c \\
-Y^{-1}+c \\
-\sqrt{1-c^{2}} \cot Y+c
\end{array} e^{2 U}= \begin{cases}\left(c^{2}-1\right) \sinh ^{-2} Y & c^{2}>1 \\
Y^{-2} & c^{2}=1 \\
\left(1-c^{2}\right) \sin ^{-2} Y & c^{2}<1\end{cases}\right.
$$

A similar ansatz can be applied in the irrotational formulation of static axisymmetric electrovacs: 


$$
\eta=\eta(\Psi)
$$

The equations that this ansatz yields are entirely similar to those of the Weyl ansatz:

$$
\begin{gathered}
\ddot{\eta}(\Psi)=0 \Rightarrow \rho^{2} e^{-2 U}=c^{\prime}+2 c \Psi-\Psi^{2} \\
d * d \Psi+b \wedge * d \Psi=\frac{2 c-2 \Psi}{c^{\prime}+2 c \Psi-\Psi^{2}} d \Psi \wedge * d \Psi
\end{gathered}
$$

For physical reasons we want $c^{\prime}+2 c \Psi-\Psi^{2}$ to be positive and this can only be achieved if $c^{\prime}+c^{2}>0$. With this restriction in mind the physical cases to be considered reduce to just one:

$$
\Psi=c+\sqrt{c^{\prime}+c^{2}} \tanh Y \quad \rho^{2} e^{-2 U}=\left(c^{\prime}+c^{2}\right) \cosh ^{-2} Y
$$

where $Y$ is again a solution of the reduced Laplace equation.

From the previous equation we learn that $\rho^{2} e^{-2 U}$ is upperly bounded by $c^{\prime}+c^{2}$ and hence these solutions cannot be asymptotically flat at infinity, since the norm of the rotational Killing vector is expected to diverge at infinity. This family could be used to represent an electrovac spacetime surrounded by a material source to which it should be matched.

Another ansatz that can be extended to this irrotational formalism is that of Papapetrou [13] for vacuum metrics. Following [14] we can write this ansatz as an assumption of functional dependence between the real and the imaginary parts of the Ernst potential:

$$
\varepsilon=e^{2 U}+i \chi \quad U=U(\chi)
$$

The real and imaginary parts of the Ernst equation imply:

$$
e^{4 U}=c^{\prime}+2 c \chi-\chi^{2}
$$

where $c$ and $c^{\prime}$ are constants.

$$
d * d \chi+b \wedge * d \chi=\frac{2 c-2 \chi}{c^{\prime}+2 c \chi-\chi^{2}} d \chi \wedge * d \chi
$$

which is similar to the one we have integrated for the extension of the Weyl solution (87). Therefore the twist potential has the following expression in terms of an arbitrary solution of the reduced Laplace equation:

$$
\chi=c+\sqrt{c^{\prime}+c^{2}} \tanh Y
$$

The restriction $c^{\prime}+c^{2}>0$ has to be imposed again for physical reasons. The Ernst potential takes the form: 


$$
\varepsilon=\sqrt{c^{\prime}+c^{2}}\left(\cosh ^{-1} Y+i \tanh Y\right)+i c
$$

If we apply this ansatz to the irrotational formalism, $\rho^{2} e^{-2 U}=g(\psi)$, instead of to the habitual shear-free formalism, then the equations have the same form and therefore we have a different family of solutions:

$$
\eta=\sqrt{c^{\prime}+c^{2}}\left(\cosh ^{-1} Y+i \tanh Y\right)+i c
$$

The same considerations for the asymptotic behavior of the extension of the Weyl family apply to this extension of the Papapetrou class.

\section{Diagonalization of the stress tensor}

Classical nonnull electromagnetics fields can be put in a frame where their stress tensor is diagonal by performing a Lorentz transformation. It implies taking the Poynting vector to be zero by letting the electric and magnetic field become parallel (if the Lorentz invariant $E \cdot B$ is different from zero) or by cancelling either the electric or the magnetic field (if $E \cdot B=0$ and the other Lorentz invariant $\|E\|^{2}-\|B\|^{2}$ is respectively negative or positive).

The same simplification can be applied without loss of generality in general relativity. This amounts to taking $E \wedge B=0$ in the exterior system and therefore the Einstein equations for the difference of the vorticity and the shear and for the dual of the acceleration remain as follows:

$$
\begin{gathered}
d *(w-s)+2 a \wedge * w+2(a-b) \wedge * s=0 \\
d * a+b \wedge * a+\frac{1}{2} w \wedge * w-\frac{1}{2} s \wedge * s=E \wedge * E+B \wedge * B=\bar{f} \wedge * f
\end{gathered}
$$

\section{1 'Seminull' Maxwell fields}

In this section we shall call 'seminull' the Maxwell fields that have one of the Lorentz invariants identically zero in opposition to null and generic fields. There are three different cases:

As we have already mentioned, when $E \cdot B=0$ and $E^{2}-B^{2}<0$ the equations can be expressed in a frame where $E=0$. We shall write only the relevant equations where the electromagnetic field appears: The Raychaudhuri equation (20) and the Maxwell equations:

$$
\begin{gathered}
d * a+b \wedge * a+\frac{1}{2} w \wedge * w-\frac{1}{2} s \wedge * s=B \wedge * B \\
d B-B \wedge a=0
\end{gathered}
$$




$$
\begin{gathered}
d * B-* B \wedge(b-a)=0 \\
B \wedge w=0 \\
* B \wedge s=0
\end{gathered}
$$

From the last two equations we get as a constraint that the vorticity and shear one-forms have to be orthogonal.

A similar case happens when $E \cdot B=0$ and $E^{2}-B^{2}>0$. Now it is $B$ the field that can be taken to be zero. The resulting equations are the same as 97-101 after changing $B$ for $E$.

The last case of seminull fields consists of those that have $E \cdot B \neq 0$, but $E^{2}=B^{2}$. Hence, in the frame where both fields are parallel, we have $E= \pm B$. Therefore $E$ and $B$ fulfill the same Maxwell equations, which take the form of equations 98 to 101. The Raychaudhuri equation becomes slightly different:

$$
d * a+b \wedge * a+\frac{1}{2} w \wedge * w-\frac{1}{2} s \wedge * s=2 E \wedge * E
$$

From the whole set of equations for the seminull Maxwell fields we get as a consequence that a solution for one of the cases provides also a solution for the other two by the following transformation rule:

$$
B_{E^{2}<B^{2}} \longrightarrow E_{E^{2}>B^{2}} \longrightarrow \frac{1}{2} E_{E \cdot B=0}= \pm \frac{1}{2} B_{E \cdot B=0}
$$

Although the three cases are physically different, their mathematical description is the same.

\subsection{A family of seminull electrovacs}

In order to derive new solutions of the Einstein-Maxwell equations we shall make use of an ansatz for the 'magnetic seminull' field:

We take it to be shear-free $(s=0)$ and assume the following condition on the kinematical one-forms:

$$
\frac{1}{2} w \wedge * w=B \wedge * B
$$

Due to the constraint 100, this ansatz implies that $B= \pm \frac{1}{\sqrt{2}} w$. Therefore we have two different sets of equations for the vorticity and its dual:

$$
\begin{aligned}
& d w=-(b-2 a) \wedge w \\
& d * w+2 a \wedge * w=0
\end{aligned}
$$




$$
\begin{gathered}
d w-w \wedge a=0 \\
d * w-* w \wedge(b-a)=0
\end{gathered}
$$

that will introduce a new constraint:

$$
b=3 a
$$

The integrability conditions for this constraint impose no new restriction since now the equations for the acceleration form and its dual are consistent with those governing $b$.

We already know that the equations for the one-form $b, 5$ and 21 , just introduce the Weyl coordinates $\rho$ and $z$, such that $* d \rho=d z$. Hence, the only equations that remain to be solved are 105 and 106, that can be straightforwardly integrated:

$$
\begin{gathered}
w=\rho^{-\frac{1}{3}} d A \\
* w=\rho^{-\frac{2}{3}} d \chi
\end{gathered}
$$

whose integrability condition in Weyl coordinates is:

$$
A_{\rho \rho}+\frac{1}{3 \rho} A_{\rho}+A_{z z}=0
$$

which is a well-known equation in hydrodynamics, the Tricomi equation in canonical coordinates when the parameters allow to classify it as an elliptic equation.

The twist potential can be obtained from the solutions of the Tricomi equation by quadratures:

$$
\begin{gathered}
\chi_{\rho}=\rho^{\frac{1}{3}} A_{z} \\
\chi_{z}=-\rho^{\frac{1}{3}} A_{\rho}
\end{gathered}
$$

Since $a=\frac{1}{3} d \ln \rho$, the metric can be expressed as follows in canonical coordinates:

$$
d s^{2}=-c \rho^{\frac{2}{3}}(d t-A d \phi)+c^{-1} \rho^{-\frac{2}{3}}\left[\rho^{2} d \phi^{2}+e^{2 k}\left(d \rho^{2}+d z^{2}\right)\right]
$$

$c$ being a positive constant and $k$ is obtained from the Cartan equations once the connection $\nu$ is calculated from the equations 22 and 23 . If we write the tetrad forms $\theta^{2}$ and $\theta^{3}$ as:

$$
\theta^{2}=e^{Q} d z \quad \theta^{3}=e^{Q} d \rho
$$


then the metric function $Q=k-U$ can be integrated from this set of equations:

$$
\begin{gathered}
Q_{z}=-\frac{3}{2} \rho^{\frac{1}{3}} A_{\rho} A_{z} \\
Q_{\rho}=-\frac{2}{9} \rho^{-1}-\frac{3}{4} \rho^{\frac{1}{3}}\left(A_{\rho}{ }^{2}-A_{z}{ }^{2}\right)
\end{gathered}
$$

whose integrability condition is precisely the Tricomi equation 112 .

From the expression for the dual of the magnetic one-form $* B$ we get the Faraday two-form $F$ :

$$
F= \pm \frac{1}{\sqrt{2 c}} d \chi \wedge d \phi
$$

and the magnetic field is :

$$
B= \pm \frac{1}{\sqrt{2}} e^{-Q} \rho^{-\frac{1}{3}}\left(A_{\rho} \theta^{2}+A_{z} \theta^{3}\right)
$$

From the general expression for the metric it is obvious that it is not asymptotically flat in canonical coordinates. This seems to arise from the fact that the gravitational field obtained from the $g_{00}$ component has cylindrical symmetry and therefore cannot be due to a compact source. The magnetic field, however, is not cylindrical unless we assume that the metric function $A$ does not depend on the coordinate $z$, in which case we would have an extra Killing vector $\partial_{z}$.

This family of solutions of the Einstein-Maxwell equations has got generically only two Killing vectors and Petrov type I. It is also generically nonstatic.

\section{Discussion}

In this paper it has been introduced a new formalism for studying stationary axially symmetric electrovacs making use of exterior differential systems of equations and putting the electromagnetic field into the form of an anisotropic fluid. This has been useful since we have not attached ourselves to any special coordinate system from the beginning and therefore we have been able to simplify our equations in different manners according to our needs.

This formulation of the coupled gravitational and electromagnetic fields has given results both in the interpretation and in the derivation of solutions of the Einstein-Maxwell equations. It has served to deal with sheets of dipoles as sources for the fields and, on the other hand, to extend old families of electrovacs and also to construct new ones. In particular, the result concerning the magnetic dipole sources for the fields is of great interest since they cannot be obtained by the usual formulation derived by Israel [8].

For a future work it would be interesting to extend further the applicability of the techniques developed so far for interpreting the discontinuities of the Ernst potentials so that other physical situations fit in the formalism. This 
would provide a deeper insight into general relativity and its comparison with flat-spacetime Physics.

The present work has been supported in part by DGICYT Project PB89-0142; L.F.J. is supported by a FPI Predoctoral Scholarship from Ministerio de Educación y Ciencia (Spain). The authors wish to thank L.M. González-Romero and J.A. Ruiz-Martín for valuable discussions.

\section{References}

[1] F. J. Ernst, Phys.Rev. 168, 1415 (1968)

[2] Solutions of Einstein's equations: Techniques and Results (eds.: C. Hoenselaers and W. Dietz, Springer Verlag), Berlin-New York (1984)

[3] El Escorial Summer School on Gravitation and General Relativity 1992: Rotating Objects and Other Topics (eds.: F. J. Chinea and L. M. GonzálezRomero), Springer-Verlag, Berlin-New York (1993)

[4] F. J. Chinea and L.M. González-Romero, Class. Quantum Grav. 9, 1271 (1992)

[5] L. M. González-Romero, Ph.D. thesis, Universidad Complutense de Madrid (1991) (unpublished)

[6] M. Gürses, J. Math. Phys. 18, 2356 (1977)

[7] L. Fernández-Jambrina and F.J. Chinea, Phys. Rev. Lett. 712521 (1993)

[8] W. Israel, Phys. Rev. D 2, 641 (1970)

[9] O. D. Kellogg, Foundations of Potential Theory. Dover, Nueva York.

[10] W. B. Bonnor, Z. Phys. 161, 439 (1961)

[11] W. B. Bonnor, Z. Phys. 190, 444 (1966)

[12] H. Weyl, Ann. d. Phys. 54, 117 (1917)

[13] A. Papapetrou, Ann. d. Phys. 12, 309 (1953)

[14] D. Kramer, H. Stephani, M. MacCallum, E. Herlt, Exact Solution's of Einstein's Field equations Cambridge University Press, Cambridge (1980) 